\title{
Improving activity and engagement for patients with dementia
}

\author{
In the second article of this series, Jennifer Bray and \\ colleagues present two case studies that show how hospital \\ trusts are helping staff and patients to forge connections
}

Correspondence

j.bray@worc.ac.uk

Jennifer Bray is research assistant

Simon Evans is principal research fellow and head of research

Mary Bruce is senior lecturer

Christine Carter is senior lecturer

Dawn Brooker is director of the Association for Dementia Studies

\section{Sarah Milosevic is former}

research assistant

All at University of Worcester

Rachel Thompson is professional and practice development lead for Admiral Nursing, Dementia UK and member of the Nursing Older People editorial advisory board

Louise Hutt is senior sister, Cambridge University Hospitals NHS Foundation Trust

Date of submission March 92015

Date of acceptance April 292015

\section{Peer review}

This article has been subject to double-blind review and has been checked using antiplagiarism software

\section{Author guidelines} journals.rcni.com/r/ nop-author-guidelines

\begin{abstract}
This is the second in a short series that presents case study examples of the positive work achieved by trusts that participated in the Royal College of Nursing's development programme to improve dementia care in acute hospitals. Staff often think that there is insufficient time to get to know patients and carers, especially with large and challenging workloads. Combined with a lack of activities and stimulation for patients with dementia in hospital, this can result in poor engagement and a disconnect between staff and patients. To improve these relationships and give staff more time with patients, Cambridge University Hospitals NHS Foundation Trust has introduced bay
\end{abstract}

nursing for patients with dementia, where one nurse is responsible for monitoring a bay alongside a healthcare assistant for an entire shift. Part of Betsi Cadwaladr University Health Board, Glan Clwyd Hospital in North Wales has focused on improving stimulation by creating an activity room with a specially trained activity worker, providing a relaxed and friendly setting where patients with dementia can take part in a range of activities and have lunch together.

\section{Keywords}

activity rooms, acute hospitals, bay nursing, dementia, development programme, patient engagement

CAMBRIDGE UNIVERSITY Hospitals NHS Foundation Trust in East Anglia is one of the largest in the UK, with around 1,000 beds and 7,000 staff. Although staff at the trust's Addenbrooke's Hospital had already undertaken a range of actions to improve the care they provided to patients with dementia, this work was driven forward by involvement in the RCN's development programme to improve dementia care in acute hospitals.

The introduction of bay nursing is one strand of work being carried out by staff at Addenbrooke's Hospital. In accordance with the SPACE principle of having skilled staff who are informed and have enough time to care (Thompson and Heath 2013) (Box 1), the benefits of encouraging staff to base themselves in bays has been demonstrated in other acute hospitals (Upton et al 2012, Waller and Masterson 2012).

A specialist ward for dementia and delirium care was identified as a candidate for bay nursing. At that time the ward was considered a challenging place to work, with a poor physical environment and little continuity of care. In addition, staff often responded to patients displaying unusual behaviours.

Staff sickness was also a problem, and the cost of providing one-to-one nursing care for distressed patients was high.

The senior sister hoped to improve dementia care and patient safety on the ward and thought that improving staff morale was important to achieve this, because staff were passionate about providing a high standard of care. An action plan was drawn up to identify the potential benefits of and barriers to bay nursing. It was presented to the senior team and shared with the whole ward team to make engagement and participation with its implementation easier.

Bay nursing identifies one nurse as responsible for monitoring each bay for an entire shift, generally from 7:15am to 7:45pm, alongside a healthcare assistant. These two staff members have a maximum of seven patients under their 
care at any time. To achieve this, the 27-bed ward had two beds removed, one from a male bay and one from a female bay. The extra space was put to good use by introducing a communal table into each bay.

The principles behind this approach include:

Staff are able to get to know a smaller number of patients in more depth.

- Staff can get to know family members better and be more approachable.

Patients are encouraged to take part in a range of activities in the bay around a table, including eating together and playing games.

- It enables the development of relationships, promotes continuity of care and allows staff adequate time with patients to "gather information about them as individuals and learn about their needs' (Thompson and Heath 2013).

Before bay nursing was implemented, one potential issue identified was the nursing of patients in side rooms. However, discussions with the wider team resulted in an agreement to check side rooms every 15 minutes, with this action being signed for by the staff member involved.

To support the introduction of bay nursing, attention was given to developing the skills of ward staff. Nine dementia champions were identified on the ward, including the ward clerk, and all staff have completed three workbooks to support their learning. The workbooks were developed by the South West Dementia Partnership and are designed for self-directed study. They cover theory and practical training in dementia care:

- Essential dementia awareness - dementia and person-centred approaches (Kukstas et al 2011a).

- Further knowledge in dementia (Kukstas et al 2011b), covering legislation, medication and safeguarding.

- Behaviours that challenge us (Kukstas and Bardsley 2011).

To ensure these workbooks are completed by staff and to formalise their use, they are included in staff appraisals as a set objective. The ward has also been developing its links with the hospital's dementia specialist nurse to make best use of this professional's knowledge and understanding of dementia.

The main challenge encountered when bay nursing was introduced was staff not understanding or appreciating that the bay could not be left unattended. It was reinforced to staff that if they left the bay the link with patients was lost and there was no one available to monitor patient safety or provide assistance as required. Staff had therefore to ensure that appropriate cover was in place if they needed to leave the bay for any reason. As two members of staff are allocated to each bay, this was thought not to be overly restrictive, although it can become more challenging during longer shifts.

Although it may require more planning to maintain a constant staff presence, the introduction of bay nursing has made a significant difference to patients and staff at Addenbrooke's Hospital. For example, having staff on hand can reduce the need for patients to try to mobilise unnecessarily and ensure that they receive support and assistance if they do get out of bed or move around the bay for any reason. Consequently, there has been a reduction in falls, with just one fall on the ward during the six months since bay nursing was introduced compared with nine in one month alone before its introduction.

Similarly, there have been no patients with hospital-acquired pressure ulcers on the ward since bay nursing began, and the number of patients requiring one-to-one nursing has reduced because there is a nurse present in the bay at all times. In addition, patients have reported that they 'feel safer, and more involved' in their care (Cambridge University Hospitals NHS Foundation Trust 2014).

Completed patient experience questionnaires suggest that patients recognise the benefit of bay nursing. Comments include: 'bay nursing gives staff the chance to get to know us, it's more sociable' and 'I do not need to use my buzzer as staff are always there to help me'.

In terms of improving social interaction, observations carried out during a site visit as part of an evaluation of the RCN development programme (Brooker et al 2014) suggested that patients do use the tables as intended, coming together at mealtimes and for communal activities such as playing

\section{Box 1 SPACE principles}

There are five principles for the care of people with dementia in hospital settings, developed in collaboration with people with dementia and carers.

Each principle has a set of sub-statements that can be used to assess progress and identify areas for further development. The principles are:

- Skilled staff who are informed and have enough time to care.

Partnership working with carers

Assessment and early identification of dementia.

Care plans that are person-centred

and individualised.

Environments that are dementia friendly.

(Thompson and Heath 2013) 


\section{Art \& science $\mid$ dementia series: 2}

\begin{tabular}{|c|c|c|c|c|}
\hline Ward & November 2013 & December 2013 & January 2014 & February 2014 \\
\hline$A$ & $3.09 \%$ & $4.46 \%$ & $5.05 \%$ & $5.81 \%$ \\
\hline$B$ & $4.94 \%$ & $3.08 \%$ & $2.90 \%$ & $3.94 \%$ \\
\hline$C$ & $5.71 \%$ & $7.77 \%$ & $7.27 \%$ & $10.08 \%$ \\
\hline$D$ & $1.59 \%$ & $2.97 \%$ & $4.02 \%$ & $4.18 \%$ \\
\hline $\begin{array}{l}\text { With bay } \\
\text { nursing }\end{array}$ & $2.80 \%$ & $1.83 \%$ & $0.53 \%$ & $1.39 \%$ \\
\hline
\end{tabular}

bingo, dominoes and Scrabble or doing puzzles. The effect of eating at a table was also mentioned by a patient's relative, who said: 'she is eating so well and gaining weight, something we have struggled with as a family for months'.

For staff, morale, sickness and satisfaction levels have all improved, as they are more in control of their work. Sickness levels on the ward compared with other care of the elderly wards in the hospital are shown in Table 1, where the hospital sickness target is $2.70 \%$.

In addition, staff report that they have been able to get to know patients and their families better as a result of working with smaller groups. The following informally gathered quotes from staff illustrate some of the positive aspects of this approach:

- 'Bay nursing is a really positive move. I enjoy being more person focused, knowing what I am doing as a result of getting to know my patients better. Showers and baths can be offered more frequently and patients can have the time to do things more independently - that is, patients assisted to walk to the toilet as opposed to given a commode because of time pressures.'

'Patients' dietary intake has improved, and I am sure patients are gaining weight rather than losing weight as expected when frail older people are taken into hospital. I get to know my patients and what they do and don't like to eat, and how and when they like to eat.'

'Patients are happy, relatives know they are not being left and staff are coming and going from work with a smile. There are fewer falls, fewer buzzers going off and no aggression from patients. What more can you ask for?'

Although the cost of bay nursing is higher because additional nurses had to be recruited to ensure a constant nursing presence in each bay, this has been offset, at least in part, by lower costs as a result of the reduction in one-to-one nursing. The number of 'specials' required on the ward dropped from more than 300 a month to less than 80 a month after the introduction of bay nursing (Cambridge University Hospitals NHS Foundation Trust 2014) (see Figure 1). In addition, in July 2014 the ward only had four 'specials' put out to bank staff.

These are all significant outcomes that illustrate improvements in the experience of care for people with dementia and their families. Unfortunately, the ward has faced challenges because some of its staff have been moved to support other areas of the hospital, making it impossible to implement bay nursing at times because of inadequate staffing levels. This has been disheartening for staff that are unable to fulfil their new role, which they know has been effective.

After the success on the initial ward, bay nursing is being implemented on a second ward, with the potential for wider adoption across the trust in the future. Plans are also in place to support the existing bay nursing by making the bay environments more dementia-friendly. This includes using a more neutral floor covering, having a nature theme for each bay and improving signage in consultation with carers. Other plans are also in place to further investigate the effect of bay nursing, for example, by monitoring length of stay.

Once staff understood what was expected of them in terms of not leaving the bays unattended and ensuring that side rooms were checked every 15 minutes, the introduction of the bay nursing concept was successful on the first ward. Indeed, one of the main recommendations from the senior sister who was instrumental in the work was the importance of meeting with the ward team in advance of implementing bay nursing to ensure that everyone knew what would be done and understood the rationale. This recommendation helped to get the whole team on board and gave staff the opportunity to raise any questions or make suggestions about how things could be done, thereby promoting ownership.

It was also found that thanking staff for their work and making them feel valued, as well as sharing positive results and outcomes, helped them to realise the effect of their work. As recommended by Manley and McCormack (2004), adoption of an inclusive and collaborative approach to developing practice is critical to facilitating and embedding new approaches to care.

\section{Creating an activity room}

Part of Betsi Cadwaladr University Health Board, Ysbyty Glan Clwyd is a district general hospital with more than 680 beds located in Rhyl, North Wales. Improving dementia care is high on the agenda for the hospital. A wider evaluation 
programme is also under way to assess the effect of all the dementia care work. Being part of the RCN development programme helped to give the hospital a focus for its work and has driven forward a scheme to open a dementia activity room.

Activities provide important stimulation for people with dementia. A survey carried out by the Alzheimer's Society (2009) found that more than $60 \%$ of carers were dissatisfied with available opportunities for social interaction and occupation when people with dementia were in hospital. The importance of activities is reflected in the SPACE principle (Box 1) of dementia-friendly environments, which recommends (Thompson and Heath 2013):

Adequate space and resources are available to support activity and stimulation.

- Availability of activity co-ordinators and trained volunteers to support activity and pastoral care. The activity room for patients with dementia was created between two elderly care wards in Glan Clwyd Hospital. The room is separate from the wards, providing a non-clinical environment and a more relaxed and friendly setting where patients can spend time during the day.

The room is run by an activity worker who previously worked as a healthcare support worker and has received additional dementia training. There was no funding for the activity worker role, so a ward had to reduce its number of staff to release sufficient funds. However, this was seen as worthwhile because fewer staff are required on the wards when patients spend time in the activity room.

The activity worker helps patients to get dressed on the ward in the morning and takes them to the activity room if they wish to attend. Activities are provided every day during morning and afternoon sessions, and include painting, word searches, dominoes, watching films, hand massages and manicures.

Patients join in with as many or as few of the activities as they wish. The room also provides a social area for patients and they are able to eat their lunch in the room if they prefer rather than returning to their bed, highlighting its flexible nature.

Dementia care mapping (DCM) was carried out to measure the effect of the activity room and the two elderly care wards. DCM is an observational framework that 'records quality of life and quality of care, from the perspective of the person with dementia' (Bradford Dementia Group 2010). Observations are scored to show the amount of time a person with dementia spent in different levels of wellbeing and ill-being, and reflect time

\section{Figure 1 Use of 'specials' for one-to-one nursing care}

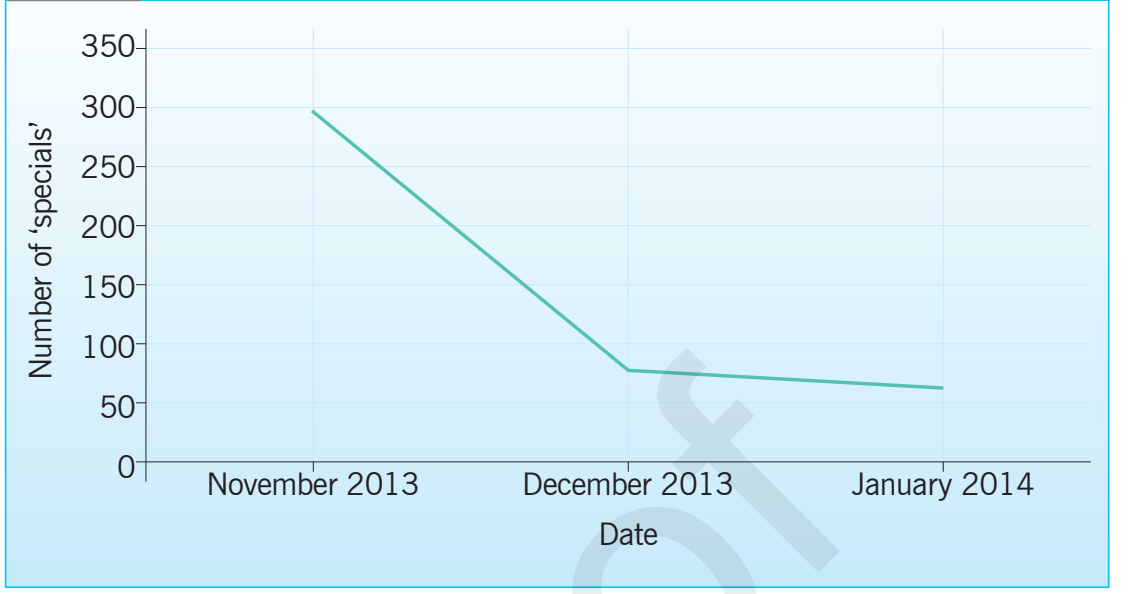

spent in positive or negative states of mood and engagement. Mean scores are calculated to provide an overall indication of wellbeing/ill-being on a scale of -5 to 5 , with higher positive scores reflecting greater wellbeing.

As can be seen from Table 2, the DCM exercise recorded a considerable difference between the mean scores for patients in the activity room and those on the two wards. The data suggest that when they were in the activity room patients with dementia were highly engaged and had a positive mood state, indicating high levels of wellbeing compared with a relatively 'neutral' experience on the wards.

In addition to DCM, information was captured as part of the wider hospital evaluation. In the six-month period after the activity room opened there were nine falls on the two wards, compared with 19 falls in the six months before the room was opened. Although focusing on a relatively short time period, this data suggest that going to the activity room and engaging with the social environment created within can potentially help to maintain or improve mobility and reduce falls for patients with dementia. The data support results from work carried out at New Cross Hospital in Wolverhampton,

Table 2 Time spent in state of wellbeing or ill-being

\begin{tabular}{|l|c|c|c|}
\hline & Ward 1 & Ward 2 & Dementia activity room \\
\hline Significant wellbeing +5 & 0 & 0 & $51 \%$ \\
\hline+3 & 0 & 0 & $43 \%$ \\
\hline+1 & $88 \%$ & $73 \%$ & $6 \%$ \\
\hline-1 & $12 \%$ & $27 \%$ & 0 \\
\hline-3 & 0 & 0 & 0 \\
\hline Significant ill-being -5 & 0 & 0 & 0 \\
\hline Mean score & 0.76 & 0.75 & 3.36 \\
\hline
\end{tabular}


where falls reduced after the introduction of a dementia-specific ward that had separate 'day rooms' and space for patients to walk around more freely (Upton et al 2012).

Awareness of the dementia activity room has spread across Glan Clwyd Hospital and staff from other wards have visited to see it for themselves. There is a certain level of healthy jealousy among staff, who ask if they can have a similar room for their wards. This indicates the positive way in which the room has been received, and the appreciation that staff have of the effect that the activity room can have on patients with dementia.

Although the benefits of having an activity room have been recognised, staff at Glan Clwyd Hospital are not assuming that their work is done. Indeed, they are already considering plans to maximise the value of the dementia activity room. One option being considered is to enable carers to use the room for confidential conversations with care co-ordinators, which could help the hospital to improve carer engagement.
The creation of a dementia activity room with a dedicated activity worker has provided a friendly and relaxed environment for patients and given them the opportunity to participate in a range of social activities. An evaluation of the effect of the room has shown that patient wellbeing is higher compared with being on a general ward, and suggests that it can have a positive effect on mobility and falls for patients with dementia.

\section{Conclusion}

The case studies presented here show that the approaches adopted by the two hospital trusts are successfully enabling staff to engage with patients in a more meaningful way. This has had a twofold impact of improving the overall care experience for the patients and helping staff to feel more positive and in control of their work. The benefits have been recognised and appreciated beyond the staff teams directly involved, and consequently the wider implementation of both approaches is being investigated.

\section{Online archive}

For related information, visit our online archive and search using the keywords

Conflict of interest None declared

\section{References}

Alzheimer's Society (2009) Counting the Cost: Caring for People with Dementia on Hospital Wards. Alzheimer's Society, London. Bradford Dementia Group (2010) Dementia Care Mapping: Principles and Practice. Third edition. University of Bradford, Bradford, Division of Dementia Studies.

Brooker D, Milosevic S, Evans S et al (2014) RCN Development Programme: Transforming Dementia Care in Hospitals Evaluation Report. University of Worcester, Worcester.

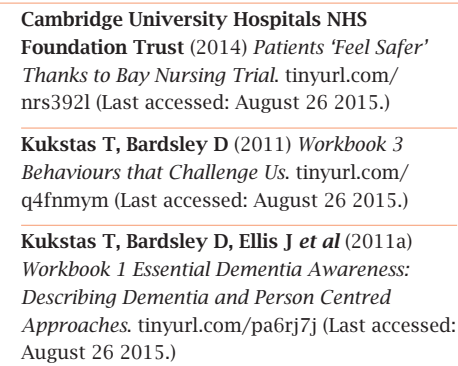

Cambridge University Hospitals NHS Foundation Trust (2014) Patients 'Feel Safer' Thanks to Bay Nursing Trial. tinyurl.com/ nrs3921 (Last accessed: August 26 2015.) Kukstas T, Bardsley D (2011) Workbook 3 Behaviours that Challenge Us. tinyurl.com/ q4fnmym (Last accessed: August 26 2015.)

Kukstas T, Bardsley D, Ellis J et al (2011a) Workbook 1 Essential Dementia Awareness: Describing Dementia and Person Centred Approaches. tinyurl.com/pa6rj7j (Last accessed: August 26 2015.)

Kukstas T, Bardsley D, Ellis J et al (2011b) Workbook 2 Further Knowledge in Dementia. tinyurl.com/petlgeq (Last accessed: August 26 2015.)

Manley K, McCormack B (2004) Practic development: purpose, methodology, facilitation and evaluation. In McCormack B, Manley K, Garbett R (Eds) Practice Developmen in Nursing. Blackwell Publishing, Oxford.

Thompson R, Heath H (2013) Dementia: Commitment to the Care of People with Dementia in Hospital Settings. RCN, London.
Upton D, Krishnan N, Bray J et al (2012) An Evaluation of Quality and Cost Effectiveness of a Newly Defined Suite of Care Interventions for Patients with Dementia and Their Carers in the Acute Hospital Setting Developed by The Royal Wolverhampton Hospitals NHS Trust, Report Phase 2 - Volume 1. Institute of Health and Society, University of Worcester.

Waller S, Masterson A (2012) Creating dementia-friendly hospitals. Health Service Journal. tinyurl.com/nz2ew8g (Last accessed: August 26 2015.) 